$\Phi=$

\title{
Pathogenicity and antibiotic resistance of Enterococcus faecalis isolated from water used in health-care centers of Ekiti State University and environ
}

\author{
Odeyemi, Adebowale Toba* and Omorovie, Paul Goodgift \\ Microbiology Department, Ekiti State University, Ado-Ekiti, Ekiti State, Nigeria \\ *Corresponding author E-mail:adebowaleodeyemi@gmail.com
}

\begin{abstract}
The quality of water samples obtained from the health-care center in the Ekiti State University and three other centers around the campus; Ekiti State University Teaching Hospital (EKSUTH), Iworoko-Ekiti health Centre (IHC) and the State Hospital, Ikole-Ekiti (SHI) were investigated by analyzing the total bacterial count using pour plate method; the incidence and antibiotic resistance of Enterococcus faecalis as water quality indicator was enumerated using selective isolation and disk diffusion method respectively. The mean TBC, TCC and TEC of all the water samples ranged from $9.1 \times 10^{2}$ to $17.4 \times 10^{3} \mathrm{CFU} / \mathrm{ml}, 4.1 \times 10^{2}$ to $5.5 \times 10^{3} \mathrm{CFU} / \mathrm{ml}$ and $0.4 \times 10^{2}$ to $0.4 \times 10^{3}$ $\mathrm{CFU} / \mathrm{ml}$ respectively. A total of 70 (32.9\%) Enterococcus faecalis were recovered from the water samples from Iworoko HC, which showed highest distribution in bore-hole and well water samples while least frequency of E. faecalis (15.7\%) was recovered from EKSU HC. However, no incidence of E. faecalis in table water obtained from all the health-care facilities. Just $35 \%$ of 20 selected E. faecalis were caseinase producers while $80 \%$ of the isolates were biofilm producers. All the isolates were resistant to cefuroxime, cefixime, augmentin and ceftazidine while only $10 \%$ of them were resistant to ofloxacin. $58.6 \%$ of the isolates showed MAR to eight (8) antibiotics with three different resistotypes while only $1.4 \%$ of them showed MAR to four (4) antibiotics with just one resistotype (CRX-CXMAUG-CAZ). Only E. faecalis 15 among the selected isolates possessed two plasmids with molecular weight of $1.415 \mathrm{bp}$ and $13.535 \mathrm{bp}$. However, consumption of contaminated water traceable to faecal sources and plasmid mediated of the causative microbes would be discussed.
\end{abstract}

Keywords: Pathogenicity; Enterococcus Faecalis; Health-Care Centers; Biofilm.

\section{Introduction}

Enterococci, which include Enterococcus faecalis, Enterococcus faecium and their varieties, are commonly found in the feces of humans and other warm blooded animals. Enterococci are members of the intestinal microbiota of healthy humans and animals and can be released into the environment, such as soil and water by human and animal fecal material (Gilmore et al., 2002). Although some strains are ubiquitous and not related to fecal pollution, Enterococci in water are an indication of fecal pollution and the possible presences of enteric pathogens.

Knowledge of Enterococci sources, fate and transport can inform the creation of models that predict Enterococci concentrations in various water sources. Measuring Enterococci on hands may be useful for understanding post collection stored water contamination in developing countries, as well as the spread of infectious disease in both developing and developed countries (Pickering et al, 2010). A methods allocates sources of Enterococci found in a surface water would be beneficial to the community of water quality managers (Jin et al., 2004).

The treatment of Enterococci infections can be difficult because Enterococcus species are intrinsically resistant to many antimicrobial agents, including cephalosporin, clindamycin, semi synthetic penicillinase-stable penicillin, and Aminoglycosides among others, and have the capacity to acquire resistance genes and mutations (Arias and Murray, 2012).
The resistance of biofilm cells formed as a result of activities of Enterococcus spp. to antimicrobial agents has serious consequences. When biofilms form on a medical device such as a ship implant, they are difficult to kill and can cause serious illness.

The aim of this project research work was to show if the presence of plasmids in bacteria is the major factor responsible for its resistance to antibiotics and basic objective of this research include: To identify the isolated organism, to determine the virulence factor of the organism, to determine the antibiotic susceptibility of the isolated organisms and the presence of plasmids in selected isolates isolated from water.

\section{Material and methods}

\subsection{Sampling site}

Water samples were taken from the health-care facility of the Ekiti State University campus, Ado-Ekiti and other three facilities around the campus which included; Ekiti State University Teaching Hospital, Ado-Ekiti; Iworoko-Ekiti health Centre and State Hospital, Ikole-Ekiti. 


\subsection{Collection of water samples}

Water samples from different sources such as well water, borehole water, chlorinated hospital water and bottled water available for use within each of these health-care centers' environments were collected aseptically using $250 \mathrm{ml}$ sterile sampling bottles, transported immediately in ice-bag to the Microbiology Laboratory of Ekiti State University, Ado-Ekiti for bacteriological analysis within $2 \mathrm{~h}$ of sample collection.

\subsection{Bacteriological analysis}

Ten-fold serial dilution was carried out on the water samples collected and $1 \mathrm{ml}$ of the water sample were inoculated on $\mathrm{Nu}$ trient agar, MacConkey agar and Bile Esculin agar using pour plating technique and incubated at $37^{\circ} \mathrm{C}$ in an incubator for 24 h invertedly according to Olutiola et al., 2000. Pure bacterial strains were isolated by streaking on freshly prepared media. Pathogenic factors of the isolated microbes were also determined through biofilm and caseinase production according to Jamal and David (2007).

\subsubsection{Antibiotics susceptibility test}

The bacterial suspension was incubated at ambient temperature until the turbidity of the suspensions was corresponding with McFarland Turbidity Standard of 0.5, which is equivalent of $10^{8} \mathrm{CFU} / \mathrm{ml}$. They were subjected to antibiotic sensitivity tests using the disc diffusion method for each of the isolate as described by Cheesbrough (2006). The antibiotics used were

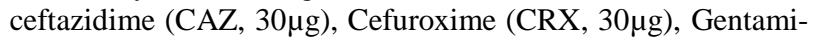

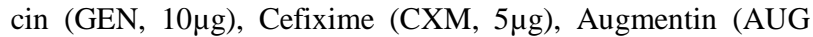

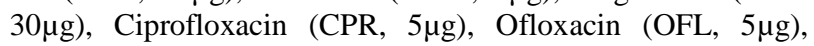
Erythromycin (ERY, $5 \mu \mathrm{g}$ ). The diameter of zone of the clearance/inhibition including the diameter of the disk was measured to the nearest whole millimeter and interpreted on the basis of CLSI (2005) guidelines.

\subsubsection{Plasmid extraction and profiling}

TENS (Tris- 25mM, Ethyl-dimethyl tetra-amine; EDTA$10 \mathrm{mM}$, Sodium hydroxide; $\mathrm{NaOH} 0.1 \mathrm{~N}$ and Sodium dodecyl sulphate; SDS 0.5\%) protocol described by Liu et al., (1995) was employed in plasmid extraction. The extracted plasmid DNA was electrophoresed on $0.8 \%$ agarose gel stained with ethidium bromide and visualized by UV-transillumination according to Robins-Browne et al., (2004).

\section{Results}

The mean total bacterial count (TBC), total coliform count (TCC) and total Enterococcus count (TEC) of water obtained from EKSU health centre (EKSU HC) ranged from $12.4 \times 10^{2}$ to $7.5 \times 10^{3} \mathrm{CFU} / \mathrm{ml}, 4.3 \times 10^{2}$ to $3.3 \times 10^{3} \mathrm{CFU} / \mathrm{ml}$ and $0.7 \times$ $10^{2}$ to $0.4 \times 10^{3} \mathrm{CFU} / \mathrm{ml}$ respectively. The same mean estimations (TBC, TCC and TEC) obtained for Iworoko health centre (IHC) ranged from $9.1 \times 10^{2}$ to $6.4 \times 10^{3} \mathrm{CFU} / \mathrm{ml}, 8.9 \times 10^{2}$ to $5.5 \times 10^{3} \mathrm{CFU} / \mathrm{ml}$ and $0.6 \times 10^{2}$ to $0.3 \times 10^{3} \mathrm{CFU} / \mathrm{ml}$ respectively. The mean values (TBC, TCC and TEC) obtained for Ekiti State teaching hospital (EKSUTH) ranged from $20.1 \mathrm{x}$ $10^{2}$ to $17.4 \times 10^{3} \mathrm{CFU} / \mathrm{ml}, 4.1 \times 10^{2}$ to $3.1 \times 10^{3} \mathrm{CFU} / \mathrm{ml}$ and $0.7 \times 10^{2}$ to $0.3 \times 10^{3} \mathrm{CFU} / \mathrm{ml}$ respectively. While mean TBC, TCC and TEC obtained for State hospital Ikole (SH.I) ranged from $11.1 \times 10^{2}$ to $9.5 \times 10^{3} \mathrm{CFU} / \mathrm{ml}, 6.1 \times 10^{2}$ to $3.2 \times 10^{3}$ $\mathrm{CFU} / \mathrm{ml}$ and $0.4 \times 10^{2}$ to $0.2 \times 10^{3} \mathrm{CFU} / \mathrm{ml}$ respectively (Table 1).

A total of 70 species of Enterococcus faecalis were recovered from the water samples with the highest frequency (32.9\%) obtained from Iworoko health centre (IHC), with highest distribution in bore-hole and well water samples. EKSUTH and SH.I comprised the same frequency of E. faecalis (25.7\%) with highest distribution from well water at State Hospital, Ikole. Meanwhile, least frequency of E. faecalis (15.7\%) was obtained from EKSU HC. There was no incidence of E. faecalis in table water obtained from all the health-care facilities (Table 2).

Among 20 selected Enterococcus faecalis isolated from water samples of the health-care facilities $35 \%$ were revealed to be caseinase producers while $80 \%$ of the isolates showed ability to produce biofilm (Table 3 ).

About $10 \%$ of isolated Enterococcus faecalis were able to resist the effect of ofloxacin. All the isolates (100\%) were resistant to cefuroxime, cefixime, augmentin and ceftazidine while very high percent (significantly above average) of the test $E$. faecalis showed resistance to gentamycin (70\%), ciprofloxacin (99\%) and erythromycin (80\%) (Table 4).

All the isolates exhibited multiple antibiotics resistance to at least four (4) of the test antibiotic with varying resistotype (combination). Highest percentage $(58.6 \%)$ of the isolates showed resistance to 8 antibiotics with 3 different resistotypes; followed by $22.9 \%$ of the isolates which were resistant to six (6) antibiotics with two (2) resistotypes. About $11.4 \%$ and $7.1 \%$ of the isolates were separately resistant to five (5) and eight (8) antibiotics with one resistotype each; and only $1.4 \%$ of the isolated E. faecalis showed resistance to four (4) antibiotics with just one resistotype (CRX-CXM-AUG-CAZ) (Table $5)$.

Out of five selected MAR Enterococcus faecalis recovered from the water samples, only E. faecalis 15 possessed two plasmids with molecular weights of $1.415 \mathrm{bp}$ and $13.535 \mathrm{bp}$ as depicted in Figure 1. 
Table 1: Microbial Load of Water Samples from Health-Care Facilities

\begin{tabular}{|c|c|c|c|c|c|c|}
\hline Sources of samples & $\begin{array}{l}\text { Total b } \\
\text { TBC } \\
10^{3}\end{array}$ & $10^{4}$ & $\begin{array}{l}\text { TCC } \\
10^{3}\end{array}$ & $10^{4}$ & $\begin{array}{l}\text { TEC } \\
10^{3}\end{array}$ & $10^{4}$ \\
\hline \multicolumn{7}{|l|}{ EKSU HC } \\
\hline A & 37 & 30 & 24 & 20 & 3 & 2 \\
\hline $\mathrm{B}$ & 25 & 19 & 29 & 18 & 5 & 3 \\
\hline $\mathrm{C}$ & 5 & 3 & 2 & 0 & 0 & 0 \\
\hline $\mathrm{D}$ & 180 & 98 & 30 & 28 & 5 & 3 \\
\hline Mean & 123.5 & 75 & 42.5 & 33 & 6.5 & 4 \\
\hline \multicolumn{7}{|l|}{ IWOROKO HC } \\
\hline A & 53 & 41 & 70 & 60 & 3 & 2 \\
\hline $\mathrm{B}$ & 24 & 16 & 80 & 30 & 4 & 2 \\
\hline $\mathrm{C}$ & 2 & 0 & 3 & 1 & 0 & 0 \\
\hline $\mathrm{D}$ & 103 & 71 & 25 & 18 & 5 & 2 \\
\hline Mean & 91 & 64 & 89 & 54.5 & 6 & 3 \\
\hline \multicolumn{7}{|l|}{ EKSUTH } \\
\hline A & 16 & 51 & 20 & 16 & 3 & 2 \\
\hline B & 205 & 165 & 30 & 25 & 2 & 1 \\
\hline $\mathrm{C}$ & 1 & 0 & 3 & 1 & 0 & 0 \\
\hline D & 180 & 132 & 29 & 20 & 8 & 3 \\
\hline Mean & 201 & 174 & 41 & 31 & 6.5 & 3 \\
\hline \multicolumn{7}{|l|}{ SH.I } \\
\hline A & 45 & 31 & 10 & 4 & 3 & 1 \\
\hline B & 49 & 38 & 30 & 20 & 3 & 2 \\
\hline C & 2 & 1 & 1 & 0 & 0 & 0 \\
\hline D & 126 & 119 & 80 & 40 & 2 & 1 \\
\hline Mean & 111 & 94.5 & 60.5 & 32 & 4 & 2 \\
\hline
\end{tabular}

Keys: $\mathrm{TBC}=$ Total Bacteria Count

$\mathrm{TCC}=$ Total Coliform Count

TEC $=$ Total Enterococcus Count

SH.I= State Hospital, Ikole

IWOROKO HC= Iworoko-Ekiti, Health center

EKSU HC= Ekiti State University, Health center

EKSUTH= Ekiti State Teaching Hospital

Table 2: Distribution of Enterococcus Faecalis in Water Samples from Water Samples in Health-Care Facilities

\begin{tabular}{llllll}
\hline \multirow{2}{*}{ Sources of sample } & \multicolumn{2}{l}{ Number of distribution per water samples } & \multicolumn{2}{c}{$\begin{array}{l}\text { Percentage Distri- } \\
\text { bution }\end{array}$} \\
\hline EKSU HC & Bore-Hole & Well water & Chlorinated HW & Bottle water & $13.7 \%$ \\
IWOROKO HC & 3 & 5 & 3 & ND & 11 \\
EKSUTH & 10 & 10 & 3 & ND & 23 \\
SH.I & 7 & 7 & 4 & ND & 18 \\
Total & 4 & 12 & 2 & ND & 18 \\
\hline
\end{tabular}

Keys: HW= Hospital water

SH.I= State Hospital, Ikole

IWOROKO HC= Iworoko-Ekiti, Health center

EKSU HC= Ekiti State University, Health center

EKSUTH= Ekiti State Teaching Hospital

$\mathrm{ND}=$ Not Detected

Table 3: Biofilm and Caseinase Production by E. Faecalis Isolated from Water Samples in Health-Care Facilities

\begin{tabular}{|c|c|c|c|}
\hline \multirow{2}{*}{$\mathrm{S} / \mathrm{N}$} & \multirow{2}{*}{ Isolates } & \multicolumn{2}{|l|}{ Pathogenic factors } \\
\hline & & Caseinaseprodudtion & Biofilm production \\
\hline 1 & E.feacalis 18 & + & + \\
\hline 2 & E.feacalis37 & - & + \\
\hline 3 & E.feacalis 19 & - & + \\
\hline 4 & E.feacalis 16 & - & + \\
\hline 5 & E.feacalis 17 & + & + \\
\hline 6 & E.feacalis 36 & + & + \\
\hline 7 & E.feacalis 9 & + & + \\
\hline 8 & E.feacalis 10 & - & + \\
\hline 9 & E.feacalis 6 & - & + \\
\hline 10 & E.feacalis 60 & + & + \\
\hline 11 & E.feacalis 70 & + & + \\
\hline 12 & E.feacalis 11 & + & - \\
\hline 13 & E.feacalis 35 & - & + \\
\hline 14 & E.feacalis 14 & - & + \\
\hline 15 & E.feacalis 41 & - & - \\
\hline 16 & E.feacalis 55 & - & + \\
\hline 17 & E.feacalis 49 & - & + \\
\hline 18 & E.feacalis 43 & - & + \\
\hline 19 & E.feacalis 45 & - & - \\
\hline \multirow[t]{2}{*}{20} & E.feacalis 47 & - & - \\
\hline & Number of positive isolates (\%) & $7(35)$ & $16(80)$ \\
\hline
\end{tabular}

Keys: + means Positive Production of biofilm and caseinase.

- means Negative Production of biofilm and caseinase. 
Table 4: Antibiotics Susceptibility among Enterococcus Faecalis Isolated from Water Samples in Health-Care Facilities

\begin{tabular}{|c|c|c|c|c|c|c|c|c|c|}
\hline Isolates & $\begin{array}{l}\text { Antibiotics } \\
\text { GEN }\end{array}$ & CPR & CRX & ERY & CXM & OFL & AUG & CAZ & Phenotype of resistant pattern \\
\hline 1 & $\mathrm{R}$ & $\mathrm{R}$ & $\mathrm{R}$ & $\mathrm{R}$ & $\mathrm{R}$ & $\mathrm{R}$ & $\mathrm{R}$ & $\mathrm{R}$ & GEN,CPR,CRX,ERY,CXC,OFL,AUG,CAZ \\
\hline 2 & $\mathrm{R}$ & $\mathrm{R}$ & $\mathrm{R}$ & $\mathrm{S}$ & $\mathrm{R}$ & I & $\mathrm{R}$ & $\mathrm{R}$ & GEN,CPR,CRX,CXM,AUG,CAZ \\
\hline 3 & I & I & $\mathrm{R}$ & I & $\mathrm{R}$ & $\mathrm{S}$ & $\mathrm{R}$ & $\mathrm{R}$ & CRX,CXM,AUG,CAZ \\
\hline 4 & $\mathrm{R}$ & $\mathrm{R}$ & $\mathrm{R}$ & $\mathrm{R}$ & $\mathrm{R}$ & $\mathrm{R}$ & $\mathrm{R}$ & $\mathrm{R}$ & GEN,CPR,CRX,ERY,CXM,OFL,AUG,CAZ \\
\hline 5 & $\mathrm{R}$ & $\mathrm{R}$ & $\mathrm{R}$ & I & $\mathrm{R}$ & $\mathrm{R}$ & $\mathrm{R}$ & $\mathrm{R}$ & GEN,CPR,CRX,CXM,OFL,AUG,CAZ \\
\hline 6 & $\mathrm{R}$ & $\mathrm{R}$ & $\mathrm{R}$ & I & $\mathrm{R}$ & $\mathrm{I}$ & $\mathrm{R}$ & $\mathrm{R}$ & GEN,CPR,CRX,CXM,AUG,CAZ \\
\hline 7 & $\mathrm{R}$ & $\mathrm{R}$ & $\mathrm{R}$ & $\mathrm{R}$ & $\mathrm{R}$ & $\mathrm{S}$ & $\mathrm{R}$ & $\mathrm{R}$ & GEN,CPR,CRX,ERY,CXM,AUG,CAZ \\
\hline 8 & $\mathrm{R}$ & $\mathrm{R}$ & $\mathrm{R}$ & $\mathrm{R}$ & $\mathrm{R}$ & $\mathrm{S}$ & $\mathrm{R}$ & $\mathrm{R}$ & GEN,CPR,CRX,ERY,CXM,AUG,CAZ \\
\hline 9 & I & $\mathrm{R}$ & $\mathrm{R}$ & $\mathrm{R}$ & $\mathrm{R}$ & I & $\mathrm{R}$ & $\mathrm{R}$ & CPR,CRX,CXM,AUG,CAZ \\
\hline 10 & I & $\mathrm{R}$ & $\mathrm{R}$ & I & $\mathrm{R}$ & $\mathrm{S}$ & $\mathrm{R}$ & $\mathrm{R}$ & CPR,CRX,CXM,AUG,CAZ \\
\hline 11 & $\mathrm{R}$ & $\mathrm{R}$ & $\mathrm{R}$ & I & $\mathrm{R}$ & $\mathrm{R}$ & $\mathrm{R}$ & $\mathrm{R}$ & GEN,CPR,CRX,CXM,OFL,AUG,CAZ \\
\hline 12 & I & $\mathrm{R}$ & $\mathrm{R}$ & $\mathrm{R}$ & $\mathrm{R}$ & $\mathrm{I}$ & $\mathrm{R}$ & $\mathrm{R}$ & CPR,CRX,ERY,CXM,AUG,CAZ \\
\hline 13 & $\mathrm{R}$ & $\mathrm{R}$ & $\mathrm{R}$ & $\mathrm{R}$ & $\mathrm{R}$ & I & $\mathrm{R}$ & $\mathrm{R}$ & GEN,CPR,CRX,ERY,CXM,AUG,CAZ \\
\hline 14 & $\mathrm{R}$ & $\mathrm{R}$ & $\mathrm{R}$ & $\mathrm{S}$ & $\mathrm{R}$ & $\mathrm{R}$ & $\mathrm{R}$ & $\mathrm{R}$ & GEN,CPR,CRX,CXM,OFL,AUG,CAZ \\
\hline 15 & $\mathrm{R}$ & $\mathrm{R}$ & $\mathrm{R}$ & $\mathrm{R}$ & $\mathrm{R}$ & $\mathrm{R}$ & $\mathrm{R}$ & $\mathrm{R}$ & GEN,CPR,CRX,ERY,CXM,OFL,AUG,CAZ \\
\hline 16 & $\mathrm{~S}$ & $\mathrm{R}$ & $\mathrm{R}$ & I & $\mathrm{R}$ & $\mathrm{S}$ & $\mathrm{R}$ & $\mathrm{R}$ & CPR,CXR,CXM,AUG,CAZ \\
\hline 17 & $\mathrm{R}$ & $\mathrm{R}$ & $\mathrm{R}$ & $\mathrm{R}$ & $\mathrm{R}$ & I & $\mathrm{R}$ & $\mathrm{R}$ & GEN,CPR,CRX,ERY,CXM,AUG,CAZ \\
\hline 19 & $\mathrm{R}$ & $\mathrm{R}$ & $\mathrm{R}$ & $\mathrm{R}$ & $\mathrm{R}$ & $\mathrm{S}$ & $\mathrm{R}$ & $\mathrm{R}$ & GEN,CPR,CRX,ERY,CXM,AUG,CAZ \\
\hline 20 & $\mathrm{R}$ & $\mathrm{R}$ & $\mathrm{R}$ & $\mathrm{R}$ & $\mathrm{R}$ & $\mathrm{S}$ & $\mathrm{R}$ & $\mathrm{R}$ & GEN,CPR,CRX,ERY,CXM,AUG,CAZ \\
\hline 21 & $\mathrm{R}$ & $\mathrm{R}$ & $\mathrm{R}$ & $\mathrm{R}$ & $\mathrm{R}$ & $\mathrm{R}$ & $\mathrm{R}$ & $\mathrm{R}$ & GEN,CPR,CRX,ERY,CXM,OFL,AUG,CAZ \\
\hline 22 & $\mathrm{R}$ & $\mathrm{R}$ & $\mathrm{R}$ & $\mathrm{R}$ & $\mathrm{R}$ & $\mathrm{I}$ & $\mathrm{R}$ & $\mathrm{R}$ & GEN,CPR,CRX,ERY,CXM,AUG,CAZ \\
\hline 23 & I & $\mathrm{R}$ & $\mathrm{R}$ & I & $\mathrm{R}$ & $\mathrm{S}$ & $\mathrm{R}$ & $\mathrm{R}$ & CPR,CRX,CXM,AUG,CAZ \\
\hline 24 & $\mathrm{R}$ & $\mathrm{R}$ & $\mathrm{R}$ & $\mathrm{R}$ & $\mathrm{R}$ & I & $\mathrm{R}$ & $\mathrm{R}$ & GEN,CPR,CRX,ERY,CXM,AUG,CAZ \\
\hline 25 & $\mathrm{R}$ & $\mathrm{R}$ & $\mathrm{R}$ & $\mathrm{I}$ & $\mathrm{R}$ & $\mathrm{S}$ & $\mathrm{R}$ & $\mathrm{R}$ & GEN,CPR,CRX,CXM,AUG,CAZ \\
\hline 26 & I & $\mathrm{R}$ & $\mathrm{R}$ & $\mathrm{R}$ & $\mathrm{R}$ & $\mathrm{S}$ & $\mathrm{R}$ & $\mathrm{R}$ & CPR,CRX,ERY,CXM,AUG,CAZ \\
\hline 27 & I & $\mathrm{R}$ & $\mathrm{R}$ & $\mathrm{I}$ & $\mathrm{R}$ & $\mathrm{S}$ & $\mathrm{R}$ & $\mathrm{R}$ & CPR,CRX,CXC,AUG,CAZ \\
\hline 28 & $\mathrm{~S}$ & $\mathrm{R}$ & $\mathrm{R}$ & I & $\mathrm{R}$ & $\mathrm{S}$ & $\mathrm{R}$ & $\mathrm{R}$ & CPR,CRX,ERY,CXM,AUD,CAZ \\
\hline 29 & $\mathrm{R}$ & $\mathrm{R}$ & $\mathrm{R}$ & $\mathrm{R}$ & $\mathrm{R}$ & $\mathrm{S}$ & $\mathrm{R}$ & $\mathrm{R}$ & GEN,CPR,CRX,ERY,CXM,AUG,CAZ \\
\hline 30 & $\mathrm{R}$ & $\mathrm{R}$ & $\mathrm{R}$ & $\mathrm{R}$ & $\mathrm{R}$ & I & $\mathrm{R}$ & $\mathrm{R}$ & GEN,CPR,CRX,ERY,CXM,AUG,CAZ \\
\hline 31 & I & $\mathrm{R}$ & $\mathrm{R}$ & $\mathrm{R}$ & $\mathrm{R}$ & $\mathrm{S}$ & $\mathrm{R}$ & $\mathrm{R}$ & CPR,CRX,ERY,CXM,AUG,CAZ \\
\hline 32 & I & $\mathrm{R}$ & $\mathrm{R}$ & I & $\mathrm{R}$ & $\mathrm{S}$ & $\mathrm{R}$ & $\mathrm{R}$ & CPR,CRX,CXM,AUG,CAZ \\
\hline 33 & $\mathrm{~S}$ & $\mathrm{R}$ & $\mathrm{R}$ & $\mathrm{R}$ & $\mathrm{R}$ & $\mathrm{S}$ & $\mathrm{R}$ & $\mathrm{R}$ & CPR,CRX,ERY,CXM,AUG,CAZ \\
\hline 34 & $\mathrm{R}$ & $\mathrm{R}$ & $\mathrm{R}$ & $\mathrm{R}$ & $\mathrm{R}$ & $\mathrm{S}$ & $\mathrm{R}$ & $\mathrm{R}$ & GEN,CPR,CRX,ERY,CXM,AUG,CAZ \\
\hline 35 & I & $\mathrm{R}$ & $\mathrm{R}$ & $\mathrm{I}$ & $\mathrm{R}$ & $\mathrm{S}$ & $\mathrm{R}$ & $\mathrm{R}$ & CPR,CRX,CXM,AUG,CAZ \\
\hline 36 & $\mathrm{R}$ & $\mathrm{R}$ & $\mathrm{R}$ & $\mathrm{R}$ & $\mathrm{R}$ & $\mathrm{S}$ & $\mathrm{R}$ & $\mathrm{R}$ & GEN,CPR,CRX,ERY,CXM,AUG,CAZ \\
\hline 40 & $\mathrm{R}$ & $\mathrm{R}$ & $\mathrm{R}$ & $\mathrm{R}$ & $\mathrm{R}$ & $\mathrm{S}$ & $\mathrm{R}$ & $\mathrm{R}$ & GEN.CPR,CRX,ERY,CXM,AUG,CAZ \\
\hline 41 & $\mathrm{R}$ & $\mathrm{R}$ & $\mathrm{R}$ & $\mathrm{R}$ & $\mathrm{R}$ & $\mathrm{S}$ & $\mathrm{R}$ & $\mathrm{R}$ & GEN,CPR,CRX,ERY,CXM,AUG,CAZ \\
\hline 42 & I & $\mathrm{R}$ & $\mathrm{R}$ & $\mathrm{R}$ & $\mathrm{R}$ & $\mathrm{S}$ & $\mathrm{R}$ & $\mathrm{R}$ & CPR,CRX,ERY,CXM,AUG,CAZ \\
\hline 43 & $\mathrm{R}$ & $\mathrm{R}$ & $\mathrm{R}$ & $\mathrm{R}$ & $\mathrm{R}$ & $\mathrm{S}$ & $\mathrm{R}$ & $\mathrm{R}$ & GEN,CPR,CRX,ERY,CXM,AUG,CAZ \\
\hline 44 & $\mathrm{R}$ & $\mathrm{R}$ & $\mathrm{R}$ & $\mathrm{R}$ & $\mathrm{R}$ & $\mathrm{S}$ & $\mathrm{R}$ & $\mathrm{R}$ & GEN,CPR,CRX,ERY,CXM,AUG,CAZ \\
\hline 45 & $\mathrm{R}$ & $\mathrm{R}$ & $\mathrm{R}$ & $\mathrm{R}$ & $\mathrm{R}$ & $\mathrm{S}$ & $\mathrm{R}$ & $\mathrm{R}$ & GEN,CPR,CRX,ERY,CXM,AUG,CAZ \\
\hline 46 & $\mathrm{~S}$ & $\mathrm{R}$ & $\mathrm{R}$ & $\mathrm{R}$ & $\mathrm{R}$ & $\mathrm{S}$ & $\mathrm{R}$ & $\mathrm{R}$ & CPR,CRX,ERY,CXM,AUG,CAZ \\
\hline 47 & $\mathrm{R}$ & $\mathrm{R}$ & $\mathrm{R}$ & $\mathrm{R}$ & $\mathrm{R}$ & $\mathrm{S}$ & $\mathrm{R}$ & $\mathrm{R}$ & GEN,CPR,CRX,ERY,CXM,AUG,CAZ \\
\hline 48 & $\mathrm{I}$ & $\mathrm{R}$ & $\mathrm{R}$ & $\mathrm{R}$ & $\mathrm{R}$ & $\mathrm{S}$ & $\mathrm{R}$ & $\mathrm{R}$ & CPR,CRX,ERY,CXM,AUG,CAZ \\
\hline 49 & $\mathrm{R}$ & $\mathrm{R}$ & $\mathrm{R}$ & $\mathrm{R}$ & $\mathrm{R}$ & $\mathrm{S}$ & $\mathrm{R}$ & $\mathrm{R}$ & GEN,CPR,CRX,ERY,CXM,AUG,CAZ \\
\hline 50 & $\mathrm{R}$ & $\mathrm{R}$ & $\mathrm{R}$ & $\mathrm{R}$ & $\mathrm{R}$ & $\mathrm{S}$ & $\mathrm{R}$ & $\mathrm{R}$ & GEN,CPR,CRX,ERY,CXM,AUG,CAZ \\
\hline 51 & $\mathrm{R}$ & $\mathrm{R}$ & $\mathrm{R}$ & $\mathrm{R}$ & $\mathrm{R}$ & $\mathrm{S}$ & $\mathrm{R}$ & $\mathrm{R}$ & GEN,CPR,CRX,ERY,CXM,AUG,CAZ \\
\hline 52 & $\mathrm{R}$ & $\mathrm{R}$ & $\mathrm{R}$ & $\mathrm{R}$ & $\mathrm{R}$ & $\mathrm{S}$ & $\mathrm{R}$ & $\mathrm{R}$ & GEN,CPR,CRX,ERY,CXM,AUG,CAZ \\
\hline 53 & $\mathrm{I}$ & $\mathrm{R}$ & $\mathrm{R}$ & $\mathrm{R}$ & $\mathrm{R}$ & $\mathrm{S}$ & $\mathrm{R}$ & $\mathrm{R}$ & CPR,CRX,ERY,CXM,AUG,CAZ \\
\hline 54 & $\mathrm{R}$ & $\mathrm{R}$ & $\mathrm{R}$ & $\mathrm{S}$ & $\mathrm{R}$ & $\mathrm{S}$ & $\mathrm{R}$ & $\mathrm{R}$ & GEN,CPR,CRX,CXM,AUG,CAZ \\
\hline 55 & $\mathrm{R}$ & $\mathrm{R}$ & $\mathrm{R}$ & $\mathrm{R}$ & $\mathrm{R}$ & $\mathrm{S}$ & $\mathrm{R}$ & $\mathrm{R}$ & GEN,CPR,CRX,ERY,CXM,AUG,CAZ \\
\hline 56 & $\mathrm{R}$ & $\mathrm{R}$ & $\mathrm{R}$ & $\mathrm{R}$ & $\mathrm{R}$ & $\mathrm{S}$ & $\mathrm{R}$ & $\mathrm{R}$ & GEN, CPR,CRX,ERY,CXM,AUG,CAZ \\
\hline 57 & $\mathrm{R}$ & $\mathrm{R}$ & $\mathrm{R}$ & $\mathrm{R}$ & $\mathrm{R}$ & $\mathrm{S}$ & $\mathrm{R}$ & $\mathrm{R}$ & GEN,CPR,CRX,ERY,CXM,AUG,CAZ \\
\hline 58 & $\mathrm{R}$ & $\mathrm{R}$ & $\mathrm{R}$ & $\mathrm{R}$ & $\mathrm{R}$ & $\mathrm{S}$ & $\mathrm{R}$ & $\mathrm{R}$ & GEN,CPR,CRX,ERY,CXM,AUG,CAZ \\
\hline 59 & $\mathrm{R}$ & $\mathrm{R}$ & $\mathrm{R}$ & $\mathrm{I}$ & $\mathrm{R}$ & $\mathrm{S}$ & $\mathrm{R}$ & $\mathrm{R}$ & GEN,CPR,CRX,ERY,CXM,AUG,CAZ \\
\hline 60 & I & $\mathrm{R}$ & $\mathrm{R}$ & $\mathrm{R}$ & $\mathrm{R}$ & $\mathrm{S}$ & $\mathrm{R}$ & $\mathrm{R}$ & CPR,CRX,ERY,CXM,AUG,CAZ \\
\hline 61 & $\mathrm{R}$ & $\mathrm{R}$ & $\mathrm{R}$ & $\mathrm{R}$ & $\mathrm{R}$ & $\mathrm{S}$ & $\mathrm{R}$ & $\mathrm{R}$ & GEN,CPR,CRX,ERY,CXM,AUG,CAZ \\
\hline 62 & $\mathrm{R}$ & $\mathrm{R}$ & $\mathrm{R}$ & $\mathrm{R}$ & $\mathrm{R}$ & $\mathrm{S}$ & $\mathrm{R}$ & $\mathrm{R}$ & GEN,CPR,CRX,ERY,CXM,AUG,CAZ \\
\hline 63 & $\mathrm{R}$ & $\mathrm{R}$ & $\mathrm{R}$ & $\mathrm{R}$ & $\mathrm{R}$ & $\mathrm{S}$ & $\mathrm{R}$ & $\mathrm{R}$ & GEN,CPR,CRX,ERY,CXM,AUG,CAZ \\
\hline 64 & $\mathrm{R}$ & $\mathrm{R}$ & $\mathrm{R}$ & $\mathrm{R}$ & $\mathrm{R}$ & $\mathrm{S}$ & $\mathrm{R}$ & $\mathrm{R}$ & GEN,CPR,CRX,ERY,CXM,AUG,CAZ \\
\hline 65 & $\mathrm{R}$ & $\mathrm{R}$ & $\mathrm{R}$ & $\mathrm{R}$ & $\mathrm{R}$ & $\mathrm{S}$ & $\mathrm{R}$ & $\mathrm{R}$ & GEN,CPR,CRX,ERY,CXM,AUG,CAZ \\
\hline 66 & $\mathrm{R}$ & $\mathrm{R}$ & $\mathrm{R}$ & $\mathrm{R}$ & $\mathrm{R}$ & $\mathrm{S}$ & $\mathrm{R}$ & $\mathrm{R}$ & GEN,CPR,CRX,ERY,CXM,AUG,CAZ \\
\hline 67 & I & $\mathrm{R}$ & $\mathrm{R}$ & $\mathrm{R}$ & $\mathrm{R}$ & $\mathrm{S}$ & $\mathrm{R}$ & $\mathrm{R}$ & CPR,CRX,ERY,CXM,AUG,CAZ \\
\hline 68 & I & $\mathrm{R}$ & $\mathrm{R}$ & $\mathrm{R}$ & $\mathrm{R}$ & $\mathrm{S}$ & $\mathrm{R}$ & $\mathrm{R}$ & CPR,CRX,ERY,CXM,AUG,CAZ \\
\hline 69 & $\mathrm{R}$ & $\mathrm{R}$ & $\mathrm{R}$ & $\mathrm{R}$ & $\mathrm{R}$ & $\mathrm{S}$ & $\mathrm{R}$ & $\mathrm{R}$ & GEN,CPR,CRX,ERY,CXM,AUG,CAZ \\
\hline 70 & $\mathrm{R}$ & $\mathrm{R}$ & $\mathrm{R}$ & $\mathrm{R}$ & $\mathrm{R}$ & $\mathrm{S}$ & $\mathrm{R}$ & $\mathrm{R}$ & GEN,CPR,CRX,ERY,CXM,AUG,CAZ \\
\hline $\begin{array}{l}\text { Percentage } \\
\text { Resistance }\end{array}$ & $70 \%$ & $99 \%$ & $100 \%$ & $80 \%$ & $100 \%$ & $10 \%$ & $100 \%$ & $100 \%$ & \\
\hline
\end{tabular}

Keys:R- Resistance, S- Suceptibility, I- Intermediate, GEN- Gentamicin, CPR-Ciprofloxacin, CRX- Cefuroxime, ERY- Erythromycin, CXM- Cefixime, OFL- Ofloxaxin, AUG- Augmentin, CAZ- Ceftazidine 
Table 5: Multiple Antibiotic Resistance Patterns of Enterococcus Faecalis Isolated from Water Samples in Health-Care Facilities

\begin{tabular}{lll}
\hline Number of antibiotics & Resistotypes & Number of Occurrences $(\%)$ \\
\hline \multirow{2}{*}{5} & CRX-CXM-AUG-CAZ & 1 \\
& Total & $1.4)$ \\
5 & CPR-CRX-CXM-AUG-CAZ & 8 \\
& Total & $8(11.4)$ \\
6 & GEN-CPR-CRX-CXM-AUG-CAZ & 4 \\
& CPR-CRX-ERY-CXM-AUG-CAZ & 12 \\
& Total & $16(22.9)$ \\
7 & GEN-CPR-CRX-CXM-OFL-AUG-CAZ & 2 \\
& GEN-CPR-CRX-CXM-OFL-AUG-CAZ & 3 \\
& GEN-CPR-CRX-ERY-CXM-AUG-CAZ & 35 \\
& Total & $40(58.6)$ \\
& GEN-CPR-CRX-ERY-CXM-OFL-AUG-CAZ & 5 \\
& Total & $5(7.1)$ \\
& Total (\%) & $70(100)$ \\
\hline
\end{tabular}

Keys:GEN- Gentamicin, CPR-Ciprofloxacin, CRX- Cefuroxime, ERY- Erythromycin, CXM- Cefixime, OFL- Ofloxaxin, AUG- Augmentin, CAZCeftazidine

Table 6: Plasmid Profile of Selected MAR Isolates from Water Samples in Health-Care Facilities

\begin{tabular}{lllll}
\hline Isolates & \multirow{2}{*}{ Number of plasmids } & \multirow{2}{*}{ Molecular weight of plasmid (bp) } & \multicolumn{2}{l}{ Antibiotics to which isolates were resistant } \\
E. faecalis 1 & - & - & 5 & GEmbination \\
\hline E. faecalis 15 & 2 & $13.535,1.415$ & 5 & GEN-CPR-CRX-ERY-CXM-AUG-CAZ \\
E. faecalis 21 & - & - & 5 & GEN-CPR-CRX-ERY-CXM-AUG-CAZ \\
E. faecalis 4 & - & - & 5 & GEN-CPR-CRX-ERY-CXM-AUG-CAZ \\
E. faecalis 5 & - & - & 5 & GEN-CPR-CRX-ERY-CXM-AUG-CAZ \\
\hline
\end{tabular}

Keys: GEN- Gentamicin, CPR-Ciprofloxacin, CRX- Cefuroxime, ERY- Erythromycin, CXM- Cefixime, OFL- Ofloxaxin, AUG- Augmentin, CAZ-

Ceftazidine

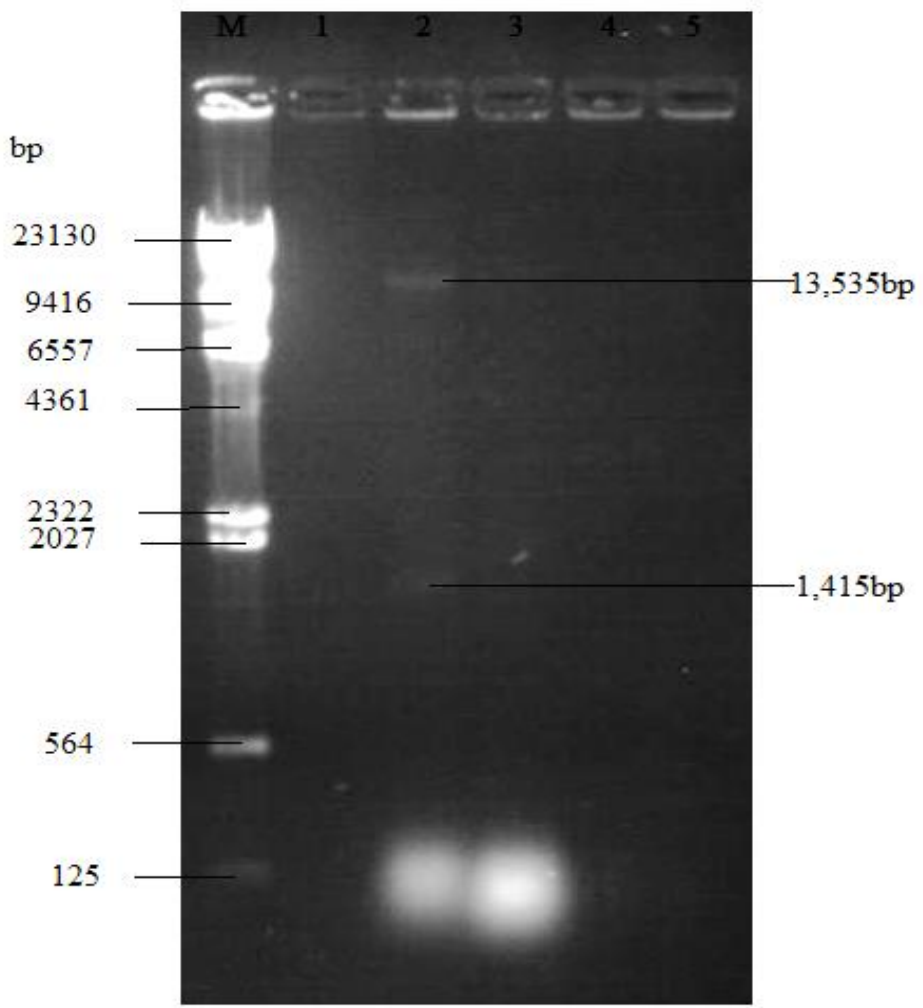

Fig. 1: Plasmid Profile of MAR Enterococcus faecalis Isolated from Water Samples in Health-Care Facilities.

\section{Discussion}

The most effective way to protect the quality of drinking water is through consistent and constant monitoring of the water supply (WHO, 2010). The quality of water is determined bacteriologically and physicochemically to ensure safe and portable water free from pathogenic microorganisms and chemical substances that are harmful to health (Shittu et al., 2008).

The mean of the total bacterial, total coliform and total enterococcal counts of the water samples were higher than the specified limit advocated by WHO, 2006. This is an indication of high microbial load which may be due to poor hygiene and sanitary condi- tions (Okonko et al., 2008). More so, Roohul-Amin et al., (2012) attributed microbial contamination of drinkable water to leakage in pipes, cross contamination with wastewater, poorly constructed well head, short distance between water supply network and sewage supply; construction of septic tanks near wells and drinking water supply lines; run-offs; infiltration of wastes and direct deposition of waste water through leakage. This evident the high microbial load encountered for the well and bore-hole water analyzed in present study.

As noted earlier, feacal indicator bacteria have been used over the years back, to assess water quality and protect humans from the myriad of enteric pathogens that are transmitted through the water 
borne route by acting as fecal indicator (Harwood et al., 2004). Fecal indicator are generally commensal habitants of the gastrointestinal tracts of many warm blooded animals and are shed in feces at high densities, thus are easily detected in contaminated water. A study had advocated that isolation of Enterococcus faecalis in water sources would be a significant factor for water quality managements (Jin et al., 2004).

Present study showed that water can serve as potential sources of health related risk factors for the consumers, simply by the presence of virulent bacterial isolates with antibiotics resistance attributes. Perhaps, Enterococci faecalis may have contaminated the water from various sources in the health-care center through different sources, as reported by Center for Environmental Health (2005). Incidence of Enterococcus faecalis in these water samples is an indication of contamination relatively of feacal origin, this is in agreement with Mathur et al. (2003) who reported that water may contain a wide range of organisms which include indigenous species, saprophytic species as well as human pathogen such as Enterococcus feacalis, Enterococcus facium, Enterococcus durans, Enterococcus avium and other species of Enterococcus. Most of the drinking water samples collected from various hospital sources was positive for Enterococcus. This could be due to many reasons. The enterococcal Strains may be more resistant to chlorination (Tree et al., 2003) or the sources of water supply to hospitals may be more polluted than open wells and bore wells. A hospital water tank has been reported as the reservoir of bacteria (Mossel and Struijk, 2002). The ubiquitous nature of the organism and its resistance to adverse environmental conditions is partly responsible for its ability to colonize different habitats and also its ability to spread easily through the water chain (Aparecida et al., 2007).

Production of pathogenic factors like biofilm by the E. faecalis isolated from water sources in health-care centers in this study is in accordance with the study of Guiton et al. (2010), which reported that biofilm production is one of the virulence factor notable of Enterococcus faecalis that contribute to its pathogenicity. Furthermore, the result obtained from this study shows that Enterococcus faecalis are multiple antibiotics resistance and the resistance to these antibiotics may be due to increase clinical use of these antibiotics and this corresponded to (Clark et al., 2011). Acquire the resistance in enterococci can occur through sporadic mutations or through acquisition of foreign genetics material. Horizontal gene exchange among Enterococci occurs through the transfer of pheromones sensitive or broad host range plasmids, or through the movement of transposons, with few exceptions, multiple plasmids and transposons can be identified in clinical strains. The result shows the presence of bacterial plasmid in Enterococci as one of the causes of their resistance to multidrug in correlation with (Hagstad et al., 2010). Factors that might lead to selective of particular groups of antibiotic resistant Enterococci in the environment are (1) antibiotic consumption by humans and animals that results in excretion of resistant bacteria, mostly in form of feces and (2) the presence of antibiotics in water that can create or maintain the selective pressure (WHO, 2004). Resistance to Erythromycin, Ciprofloxacin, Augmentin, Cefixime, Ceftazidine, Cefuroxime can be mostly explained by the presence of the classical erm(B) gene alone or combined with the efflux mef(A) gene and this correlated with (Arias et al., 2012).

According to the report of (Odeyemi et al., 2010) which stated Enterococcus have a remarkable capacity of expressing resistance to several groups of antimicrobial, thus posing a daunting challenge to the word of clinical practice as the number of therapeutic options for medical interventions are significantly reduced. Entetrococci are not pathogenic organisms in nature, they are part of the microbiota of the body, but there is infection in the body or immunocompromise patient create an opportunity of becoming harmful to the host.

\section{Conclusion and recommendations}

Present study has shown that the water samples from the healthcare facilities in the Ekiti State University and Environ are not safe for drinking due to contaminations that are traceable to faecal sources. Incidence of a noble water quality indicator, Enterococcus faecalis especially in high frequency in these water sources is however a potential health hazard. Moreover, Enterococcus faecalis recovered from this study showed the ability to develop resistance to essentially every antibiotic used against them, serving as a great threat to the community.

This study therefore recommends that future studies should further dissect the mechanism by which Enterococcus faecalis could colonize water body and should then focus on ways to reduce or prevent this colonization. Strategies, including immunological approaches, to prevent infection in the face of colonization should be explored. In addition, the elucidation of mechanisms of resistance to the newer anti-enterococcal antibiotics should yield important insights for the development of future therapeutic options. Additional new strategies, perhaps phage-based or a combined therapeutic-immunological approach should also be considered.

\section{References}

[1] Aparecida, P.F.S., Scheidegger, E.M.D., Santos, P.F., Leite, P.C and Teixeira, L.M. (2007). Antimicrobial resistance profiles of Enterococci isolated from poultry meat and pasteurized milk in Rio de Janeiro, Brazil. Mem. Inst. Oswaldo Cruz 102(7): 853-859. Available online at from: http://www.scielo.br/scielo.php?script=sci_arttext\&pid=S00740276 2007000700011\&LNG=en. [Cited 2010 Mar 16].

[2] Arias, C. A. and Murray, B. E. (2012): The rise of the Enterococcus: beyond Vancomycin resistance. Nature reviews. Microbiology, 10(4): p. 266-278.http://dx.doi.org/10.1038/nrmicro2761.

[3] Clark, C. MCGhee, P., Appelbaum, P. C. and Kosowska-Shick, K (2011): Multistep resistance development studies of ceftaroline in gram-positive and negative bacteria. AntimicrobiolAgentsChemothers. 55:2344-51.http://dx.doi.org/10.1128/AAC.01602-10.

[4] Clinica and Laboratory Standard Institute.CLSI. (2005): Performance standard for antimicrobial susceptibility testing $15^{\text {th }}$ informational supplement M100-S15.

[5] Cheesbrough, M. (2006). District laboratory Practice in Tropical Countries. Part 2. Cambridge University Press. pp. 143 157.http://dx.doi.org/10.1017/cbo9780511543470.

[6]

Gilmore, M. S., Coburn, P. S., Nallapareddy, S. R., and Murray, B. E. (2002). Enterococcal virulence. InM.S.Gilmore (Ed.), the enterococci: Pathogenesis, molecularbiology and antibiotic resistance (pp. 301-354). Washington, DC: American Society for Microbiology Press.http://dx.doi.org/10.1128/9781555817923.ch8.

[7] Guiton, C. S. Hung, L.E., Hancock, M. G. and Caparon, S.J. (2010): HultgrenEnterococcal Biofilm formation and virulence in an optimized murine model of foreign body-associated urinary tract infections. Infect. Immun., 78 (10). pp. 4166 4175.http://dx.doi.org/10.1128/IAI.00711-10.

[8] Harwood, V.J, et al. (2004). Molecular confirmation of Enterococcus faecalis and E. faecium from clinical, faecal and environmental sources. Lett. Appl. Microbiol. 38: 476 482.http://dx.doi.org/10.1111/j.1472-765X.2004.01518.x.

[9] Hegstad, K., Mikalsen, T., Coque, T. M., Werner, G. and Sundsfjord, A. (2010). Mobile genetic elements and their contribution to the emergence of antimicrobial resistant Enterococcus faecalis and Enterococcus faecium. ClinMicrobiol Infect, 16:541-554. http://dx.doi.org/10.1111/j.1469-0691.2010.03226.x.

[10] Jamal A. M. and David B. H. (2007). Biofilm formation by Enterococci. Journal of Medical Microbiology, 56, 15811588.http://dx.doi.org/10.1099/jmm.0.47331-0.

[11] Jin, G., Englande, A. J., Badford, H. and Jeng, H. W. (2004). Comparison of E. coli, Enterococci, and fecal coliform as indicators for brackish water quality assessment. WaterEnviron. Res. 76: 245255.http://dx.doi.org/10.2175/106143004X141807.

[12] Liu, Z. M., Tucker, A. M., Driskell, L. O. and Wood, D. O. (1995) Mariner based transposon mutagenesis of Rickettsia prowazeku. 

App
Environ. Microbiol.
$73: 6644$

6649.http://dx.doi.org/10.1128/AEM.01727-07.

[13] Mathur, P., Kapil, A., Chandra, R., Sharma, P. and Das, B. (2003). Antimicrobial resistance in Enterococcus faecalis in a tertiary care centre of northern India. Indian J. Med. Res., 118:25-8.

[14] Center for Environmental Health (2005). Coliform bacteria in drinking water supplies. New York state Department of Health, 1800-458-1158 ext. 2-7650 or wsp@ @ealth.state.ny.us.

[15] Mossel, D.A. and Struijk, C.B. (2002). Assessment of the microbia integrity, sensu G.S. Wilson, of piped and bottled drinking water in the condition as ingested. In Proceedings of the NSF International/WHO Symposium on HPC Bacteria in Drinking Water Public Health Implications? (ed. J. Bartram, J. Cotruvo and C. Fricker), pp. 363-376, NSF International, Ann Arbor, MI.

[16] Odeyemi, A. T., Dada, A. C., Ogunbanjo, O. R. and Ojo, M. A. (2010). Bacteriological, physiochemical and mineral studies on Awedele spring water and soil samples in Ado-Ekiti, Nigeria. African Journal of Environmental Science and Technology, 4(6):319327.http://dx.doi.org/10.5897/AJEST09.194.

[17] Okonko, I. O., Adejoye, O. D.,Ogunnusi, T. A., Fajobi, E. A. and Shittu, O. B. (2008). Microbiological and Physiochemical Analysis of Different Water Samples used for Domestic purposes in Abeokuta and Ojata, Lagos State, Nigeria. African Journal of Biotechnology, 7(5):617 - 621 .

[18] Olutiola, P. O., Famurewa, O. and Sonntag, H. S. (2000). An introduction to General Microbiology (A practical Approach); Measurement of microbial growth. Pp. 101-111.

[19] Pickering, A. J., Davis, J., Walters, S. P., Horak, H. M., Keymer, D. P. and Mushi, D. (2010). Hands, water and health fecal contamination in Tanzanian communities with impored, non-networked water supplies. Environ Sci Technol. 44(9):32673272.http://dx.doi.org/10.1021/es903524m.

[20] Robins-Browne, R.M., Bordun, A.M., Tauschek, M., BennettWood, V.R., Russell, J., Oppedisano, F., et al., (2004). Escherichia coli and community-acquired gastroenteritis, Melbourne, Australia. $\begin{array}{llll}\text { Emerg. } & \text { Infect. } & \text { Dis. } & 1797\end{array}$ 1805.http://dx.doi.org/10.3201/eid1010.031086.

[21] Roohul-Amin., Ali, S.S., Anwar, Z., and Khattak, J.Z.K. (2012) Microbial analysis of drinking water and water distribution system in New Urban Peshawar. Current Research Journal of Biological Sciences. 4 (6), 731-737.

[22] Shittu, O. B., Olaitan, J. O. and Amusa, T. S. (2008). Physicochemical and bacteriological analyses of water used for drinking and swimming purposes in Abeokuta, Nigeria. African Journal of Biomedical Research. 11: 285 - 290.

[23] Tree, J. A., Adams, M. R. Lees, D. N. (2003). Chlorination of indicator bacteria and viruses in primary sewage effluent, Appl. Environ. Microbiol. 69: 2038 2043.http://dx.doi.org/10.1128/AEM.69.4.2038-2043.2003.

[24] World Health Organization (2004). Guidelines for drinking water quality.3rd edition, Switzerland: WHO press pp.16, 89.

[25] World Health Organization (2006). Guidelines for Drinking Water Quality Vol.1 Geneva, Switzerland.

[26] World Health Organization (2010). Guidelines for Drinking Water Quality Vol. 3 Geneva, Switzerland. 